\title{
Coherency Identification of Generators Using a PAM Algorithm for Dynamic Reduction of Power Systems
}

\section{Gi-Chan Pyo *, Jin-Woo Park and Seung-Il Moon}

School of Electrical Engineering and Computer Science, Seoul National University, Seoul 151-742, Korea; E-Mails: jwpark@powerlab.snu.ac.kr (J.-W.P.); moonsi@plaza.snu.ac.kr (S.-I.M.)

* Author to whom correspondence should be addressed; E-Mail: draco99@powerlab.snu.ac.kr; Tel.: +82-2-886-3101.

Received: 15 September 2012; in revised form: 31 October 2012 / Accepted: 31 October 2012 / Published: 8 November 2012

\begin{abstract}
This paper presents a new coherency identification method for dynamic reduction of a power system. To achieve dynamic reduction, coherency-based equivalence techniques divide generators into groups according to coherency, and then aggregate them. In order to minimize the changes in the dynamic response of the reduced equivalent system, coherency identification of the generators should be clearly defined. The objective of the proposed coherency identification method is to determine the optimal coherent groups of generators with respect to the dynamic response, using the Partitioning Around Medoids (PAM) algorithm. For this purpose, the coherency between generators is first evaluated from the dynamic simulation time response, and in the proposed method this result is then used to define a dissimilarity index. Based on the PAM algorithm, the coherent generator groups are then determined so that the sum of the index in each group is minimized. This approach ensures that the dynamic characteristics of the original system are preserved, by providing the optimized coherency identification. To validate the effectiveness of the technique, simulated cases with an IEEE 39-bus test system are evaluated using PSS/E. The proposed method is compared with an existing coherency identification method, which uses the K-means algorithm, and is found to provide a better estimate of the original system.
\end{abstract}

Keywords: dynamic reduction; equivalent circuits; power system modeling 


\section{Introduction}

In recent years, power systems have experienced rapid changes accompanying the integration of various power electronic devices and large-scale renewable energy sources. These changes may have unexpected impacts on power system stability. To maintain the stability and improve the performance of a system, the transient stability in response to such changes should be assessed continuously. However, transient stability assessment based on real-time dynamic simulations is subject to limitations when applied to large-scale nonlinear power systems, since the momentary processing ability of a simulator is limited by the given hardware specifications. In a modern power system, it is almost impossible to study transient stability problems for the entire system because of its large size and complexity, even when expansion is considered. Dynamic reduction of a power system is a good alternative for transient analysis of the entire power system, provided it preserves the dynamic characteristics of the original system with acceptable accuracy [1-4]. When studying transient stability using detailed system models, it is a common practice to represent a large power system with some form of reduced equivalent model via dynamic reduction [5-8].

In recent decades, several dynamic reduction techniques have been developed, including coherency-based equivalence techniques, modal equivalence techniques, and slow coherency techniques (which combine the merits of both of the preceding types). Of these, the coherency-based techniques are most widely used in practical applications for nonlinear dynamic simulation because of their simplicity [1,3-9]. In a dynamic reduction procedure, a coherency-based technique defines coherency in terms of the behavior of generators during severe disturbances, divides them into groups according to coherency, and then aggregates generators in the same group as an equivalent generator [7-11]. A coherency-based dynamic reduction method ensures greater accuracy of the reduced equivalent model, provided coherency identification of the generators is clearly defined. Consequently, research to evaluate and identify coherency from the dynamic responses of generators mainly have been conducted in relation to coherency-based dynamic reduction techniques. However, relatively few grouping algorithms have been developed to determine generator groups according to coherency. Existing algorithms are also subject to some limitations when applied to the dynamic reduction of modern power systems, since the complexity of power systems has recently increased with the number of generators. The grouping algorithm proposed by Van Oirsouw [12] has several drawbacks arising from its sequential procedure, despite its advantages of speed and simple application. When this algorithm is used, the first generator group becomes the largest group in the majority of cases, and a generator can be grouped with other generators with which it is hardly coherent, since the group in which a generator is placed is determined solely by comparison with the last generator assigned to that group. On the other hand, the recently proposed coherency identification technique using the K-means algorithm solves most of the problems associated with Van Oirsouw's algorithm [13,14]. However, this approach still has some drawbacks, such that the groups are determined differently, depending on the calculation procedure and initial group. This means that the resulting coherent groups are unlikely to be the optimized case unless the calculation sequence is defined properly.

This paper proposes a new coherency identification method based on the Partitioning Around Medoids (PAM) algorithm. The PAM algorithm is a typical clustering algorithm, and like the K-means 
algorithm, it has been used in the data mining area. Compared to the K-means algorithm, this procedure provides better optimized results regardless of the calculation procedure and initial conditions, since it minimizes a cost function (a sum of dissimilarities) instead of using only the Euclidean distances between individual objects. The proposed approach evaluates the coherency among generators from the dynamic simulation time responses, and defines it as a dissimilarity index. Coherent generator groups are determined to minimize the sum of this index in each group, based on the PAM algorithm. For this purpose, the time responses of the generators are obtained by dynamic simulation using PSS/E program, and the reduced equivalent model is verified by comparing the dynamic simulation results with those of the original system.

\section{Proposed Coherency Identification Method}

As mentioned above, coherency is defined by the dynamic responses of generators during severe disturbances, such as system faults. Two generators are considered to be coherent when the phase angle difference of their terminal voltages is bounded by some specified quantity over a certain period of simulation time. The coherency condition generally simplifies to:

$$
\delta_{i}(t)-\delta_{j}(t)=\delta_{i j}(t) \leq \mathrm{const}
$$

where $\delta_{i}(t)$ is the rotor angle of the $i^{t h}$ generator and $\delta_{j}(t)$ is the rotor angle of the $j^{t h}$ generator. Consequently, coherency indicates the relative electric coupling between generators in the system and the coherent generators are expected to have similar dynamic characteristics and responses during severe disturbance, such as a fault sequence. Generators satisfying Equation (1) are then aggregated and replaced by a single equivalent generator in the dynamic reduction.

\subsection{Dissimilarity Index}

The first step in coherency identification is to define coherency from the dynamic response of the system. Conceptually, coherency is identical to the dissimilarity that is usually used in clustering algorithms, in that both indicate the relationship between objects equally well. This means that a clustering algorithm such as the PAM algorithm can be used to construct coherent groups of generators, provided coherency is defined numerically. In order to apply the PAM algorithm to coherency identification, a dissimilarity index is proposed to define the coherency of the generators in this paper. For this purpose, swing curves for all generators are obtained from the time response during a disturbance, using the PSS/E simulator. The rotor angle vector of the $i^{\text {th }}$ generator can be defined by sampling the simulation results, as follows:

$$
\delta\left(G_{i}\right)=\left(x_{1}, x_{2}, x_{3}, x_{4}, \ldots, x_{n}\right)
$$

where $x_{k}$ is the sampled rotor angle for each time interval. The normalized rotor angle vector for $i^{\text {th }}$ generator, $\delta_{N}(i)$, is then obtained from (1) via the following equation:

$$
\delta_{N}\left(G_{i}\right)=\frac{\delta\left(G_{i}\right)-\delta_{\text {init }}\left(G_{i}\right)}{\max \left\{\left|\delta\left(G_{i}\right)\right|\right\}-\min \left\{\left|\delta\left(G_{i}\right)\right|\right\}}
$$


where $\delta_{\text {init }}\left(G_{i}\right)$ is the initial rotor angle of the generator. In this equation, the dc offset is eliminated in order to remove the effect of the initial value, and the rotor angle vector is normalized by dividing by the difference between its maximum and minimum values. The dissimilarity index between two generators is determined from the normalized rotor angle vectors, as follows:

$$
D\left(G_{i}, G_{j}\right)=\left\|\delta_{N}\left(G_{i}\right)-\delta_{N}\left(G_{j}\right)\right\|_{2}
$$

Since the dissimilarity index $D\left(G_{i}, G_{j}\right)$ is defined as the Euclidian distance between the angles of two generators for a given time duration, the correlation between the generators decreases as the value of the index increases, contrary to the usual definition of coherency.

\subsection{Define the Generator Groups Using PAM Algorithm}

In next step, the coherent groups of generators are determined using the dissimilarity index with the criterions based on PAM algorithm. The proposed generator grouping algorithm consists of the following steps:

Step 1. Calculate the Dissimilarity Index (DI) table;

Step 2. Build: Determine the initial generator set $M$;

Step 3. Swap: Perform a swap test between the generators of set $M$ and the non-medoid set;

Step 4. Cluster the generators using the optimal medoid set.

In the PAM algorithm, a medoid is the object representative of a group. All objects are classified into clusters to minimize the sum of the dissimilarities between objects and medoids [15]. Accordingly, each cluster of objects, corresponding to a group of generators in the proposed approach, is determined uniquely for a given medoid set by selecting the medoid that is closest to the objects in the cluster. The optimal clusters are then obtained by examining the objective function for every feasible medoid set, and selecting the medoid set that minimizes the objective function. The proposed generator grouping algorithm uses a typical form of the PAM clustering algorithm. The generators in the target system are classified into optimal coherent groups, so that the sum of the dissimilarities between the generators and medoid of each group (i.e., the objective function) is minimized. The following subsections provide brief descriptions of each of the four steps:

(A) Calculate the Dissimilarity Index: As mentioned above, the Dissimilarity Index (DI) between generators is determined by the rotor angle vectors obtained from the dynamic time simulation, which is arranged in tabular form in this procedure. The value of the dissimilarity index of generators $i$ and $j$, $D\left(G_{i}, G_{j}\right)$ is calculated by Equation (4), and the cost function of the overall generator grouping algorithm can be defined as follows:

$$
\sum_{i=1}^{K} \sum_{j=1}^{N} D\left(G_{i}, G_{j}\right)=T C \quad \text { for } G_{i} \in M, G_{j} \in C_{i}
$$

where $K$ is the number of groups and $N$ is the number of generators in the $i^{\text {th }}$ coherent group $C_{i}$. In this equation, $M$ denotes the medoid set used in the PAM algorithm, and $G_{i}$ indicates the generator corresponding to the medoid of the $i^{\text {th }}$ coherent generator group. This cost function is calculated continuously using the DI during the overall coherency identification procedure, and the coherent 
group of each generator is determined to minimize the cost function. The cost function is referred to as $T C$ in the remainder of the paper, and the objective function of the PAM algorithm is generally defined to minimize $T C$.

(B) Build: Determine the initial medoid set M: To determine the groups of generators that minimize the cost function, an initial set of generators corresponding to the medoids is necessary. The initial value of the cost function is calculated using this generator set. Generally, the initial medoid set can be chosen arbitrarily in the PAM algorithm. In the proposed method, however, some of the initial generators are determined from existing power system information. Generators with similar controls or connected to nearby buses are normally expected to be coherent. These generators are likely to be included in the same group after identifying coherency, and one of them can be used as the representative generator of the coherent group. Therefore, if generator information (such as bus connections and control systems) is available, the calculations can be reduced by choosing the initial generator set using this information. The remaining generators are then added via the proposed algorithm, and the efficiency and convergence of the clustering algorithm are thereby improved. The detailed procedure for selecting the initial generator set is as follows:

B.1. Determine an initial user-defined medoid set $M(1 \leq n(M) \leq K)$;

B.2. Calculate $C_{k}=\sum_{\{j \neq k, j \notin M\}}\left(\min \left\{D\left(G_{j}, G_{i} \in M\right)\right\}-D\left(G_{j}, G_{k}\right)\right)$ for all non-medoid generators $k$;

B.3. Add a generator which maximizes $C_{k}$ to the medoid set $M$;

B.4. if $n(M)=K$, go to the next procedure;

if $n(M)<K$, go to step 2 and repeat step 2-3.

where $n(M)$ is the number of generators in the initial user-defined medoid set and it should be smaller than or equal to $K$, the number of groups, at the beginning of this procedure. In the step B.2., $C_{k}$ indicates notionally a possible decrease in the cost function $T C$ when generator $G_{k}$ becomes a medoid, and a generator which has a larger $C_{k}$ than the others is more likely to be included in the medoid set after identifying coherency. Therefore, $C_{k}$ is tested for all non-medoid generators in this step, and a generator which makes $C_{k}$ maximized is added to the initial medoid set $M$. This procedure is repeated until the number of generators in the initial medoid set is equal to the number of coherent groups.

(C) Swap: Perform a swap test for generators in set $M$ and the non-medoid set: Once the initial generator set $M$ has been determined, each generator in the medoid set is tested by successively swapping it with all generators in the non-medoid set. Then, the change in the cost function is checked. During this process, a non-medoid generator that makes the cost function smaller than it was just before a swap is selected to replace the medoid generator with which it was swapped. The detailed procedure for swap test is as follows:

C.1. Swap generator $G_{i}$ in the medoid set $\mathrm{M}$ with generator $G_{j}$ in the non-medoid set;

C.2. Calculate $\triangle T C_{i j}$ for all $j$;

C.3. if $\operatorname{Min}\left\{\Delta T C_{i j}\right\}<0$, generator $G_{j}$ replaces generator $G_{i}$ as a medoid; if $\left.\operatorname{Min}\left\{\Delta T C_{i j}\right\}\right) \geq 0$, go to step 5;

C.4. Select the next generator $i$ in set $M$ and repeat step 1-3;

C.5. Repeat steps $1-4$ until $\Delta T C_{i j} \geq 0$ for all $i$. 
Figure 1 illustrates these procedures conceptually. The optimal medoid set is determined when the change in the cost function $T C$ during the swap test has a nonnegative value for all generators $G_{i}$ in the medoid set.

Figure 1. The swap test for the generators in the set $M$ and the non-medoid set.
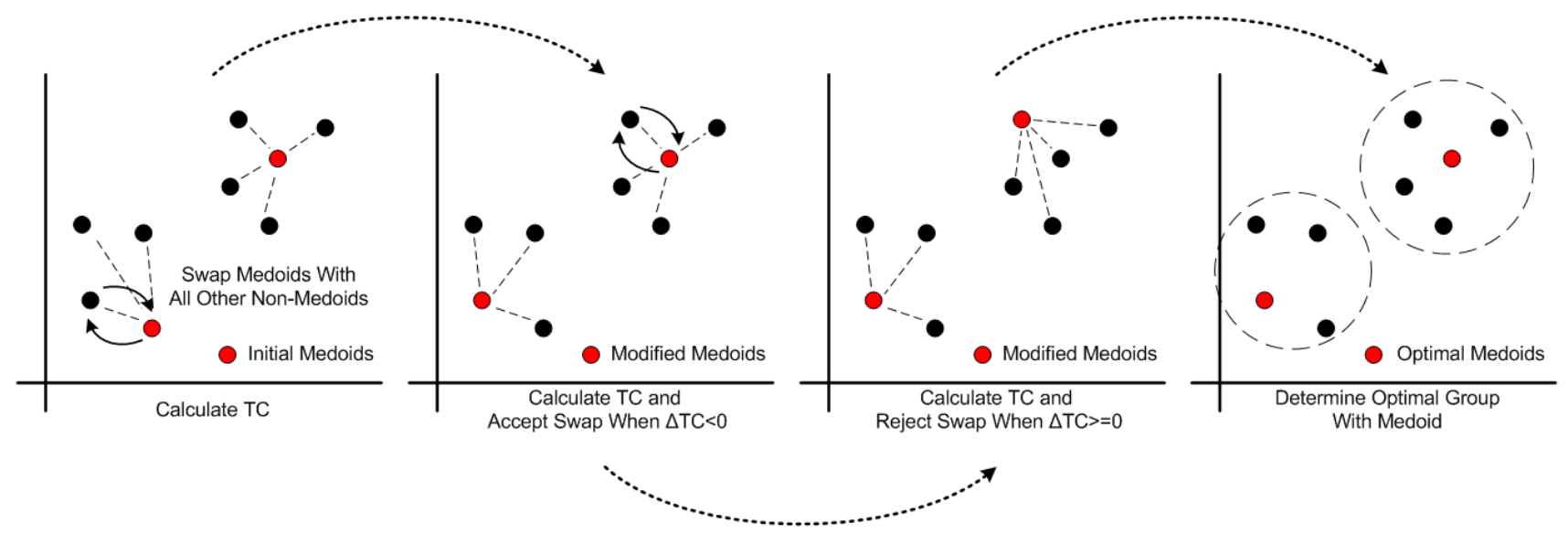

(D) Determine the groups of generators using the optimal medoids set: In this step, groups of generators are determined using the optimized medoid generator set $M$ obtained in the previous steps. The generators are divided into a given number of coherent groups by selecting the medoid that has the minimum dissimilarity index with each generator. Consequently, the optimal coherent groups are determined uniquely when the cost function is minimized. Figure 2 shows a flow chart of the overall generator grouping algorithm explained in this chapter.

Figure 2. Flow chart of the proposed algorithm.

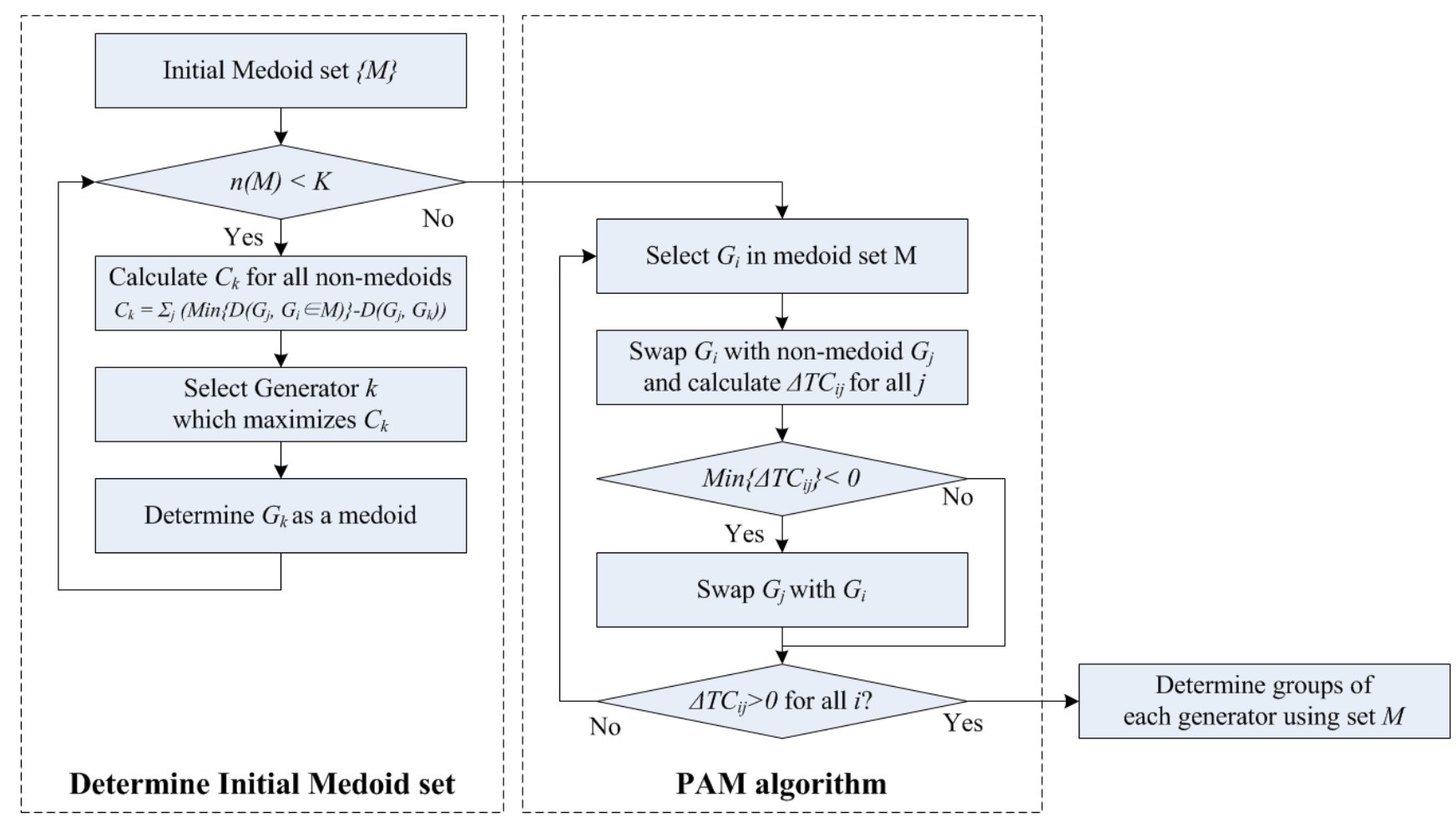


In the proposed coherency identification method, the PAM algorithm is used to determine the set of coherent generator groups. The algorithm obtains the optimized coherent groups of generators by minimizing the cost function $T C$ (the sum of the dissimilarity indices). This means that the generators in each coherent group are generators with the most similar characteristics in the transient state. Therefore, the proposed approach ensures that the generators in each group can be aggregated and replaced by an equivalent generator, while preserving the dynamic characteristics of the original system with high accuracy.

\subsection{Generator Aggregation}

Once the coherent groups have been identified, the generators in each group are aggregated and replaced by a single equivalent generator, while maintaining the steady-state power flow of the original system for the next step. A detailed generator model with an exciter and a governor is used in this process. The detailed generator aggregation procedure is as follows:

(1) Join the buses;

(2) Aggregate the static generator and load model;

(4) Aggregate the dynamic generator model;

(5) Aggregate the control units.

After each generator group has been aggregated, the modified network contains only equivalent generators that represent coherent groups and the original loads and buses. Network reduction is then performed using a static reduction method, such as the Ward-PV equivalence technique [16], if needed. This eliminates selected load nodes of unconcerned subsystems, and provides accurate results when applied to a passive network. With this coherency-based dynamic reduction of generators, a power system is reduced to a smaller equivalent system, which has the appropriate size and complexity for the practical use of transient simulators.

\section{Case study}

In order to verify its effectiveness, the proposed coherency identification method was compared with an existing method based on the K-means algorithm [13]. For this purpose, both procedures were applied to the dynamic reduction of an IEEE 39-bus test system. The PSS/E simulator program is used for the dynamic simulations, and the dynamic responses of the two equivalent reduced systems to a fault sequence are compared. Figure 3 shows a diagram of the IEEE 39-bus test system used in the case studies.

\subsection{Coherency Identification for Dynamic Reduction}

The test system was divided into internal and external systems for the dynamic reduction. Figure 4 shows the division of the system. The dynamic reduction was performed on the external system, which included six generators and 24 buses. In the external system, the load buses are to be eliminated, considering the effects at boundary buses, and the generators are to be aggregated into the given number of equivalent generators, once the coherent groups have been determined by the coherency identification method. 
Figure 3. One line diagram of the IEEE 39-bus test system.

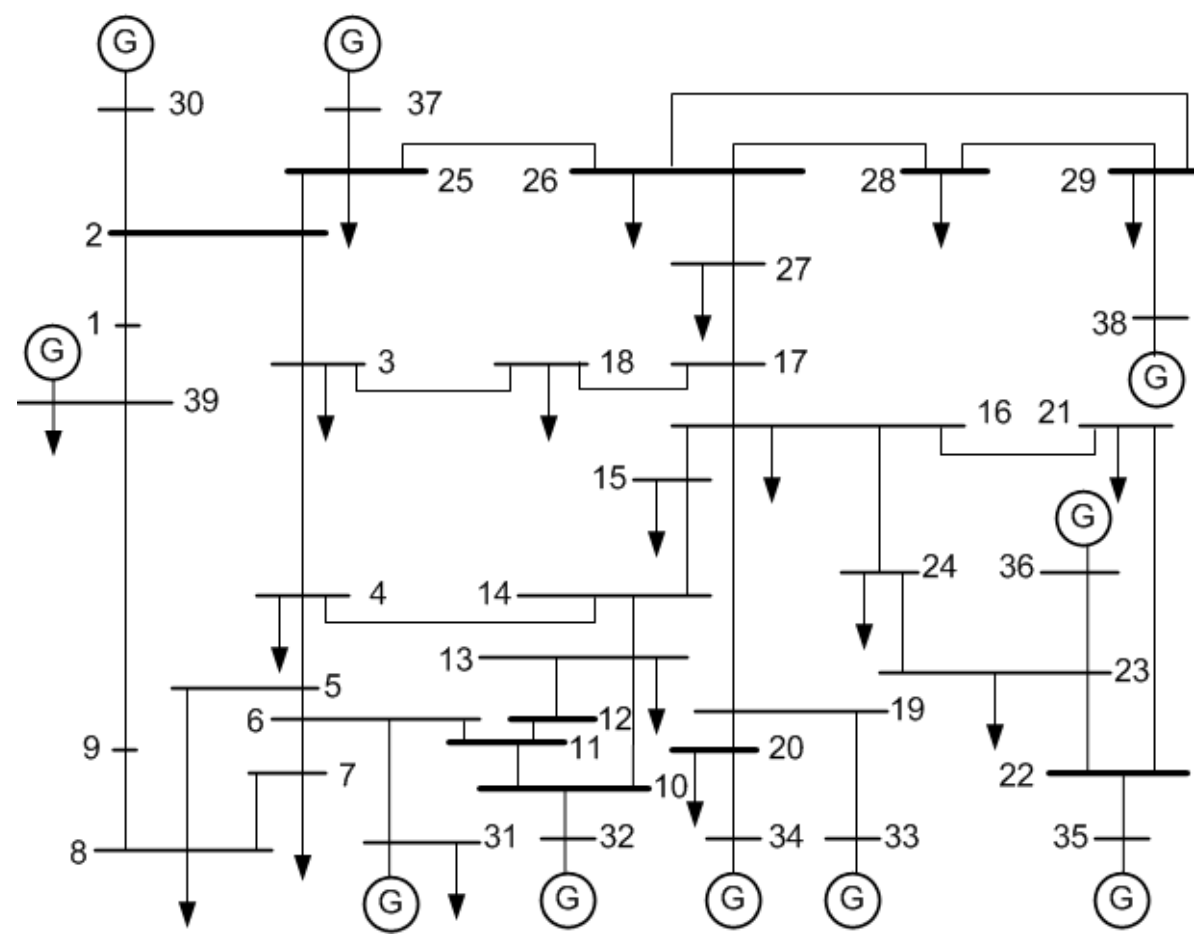

Figure 4. Division of the system into internal and external systems.

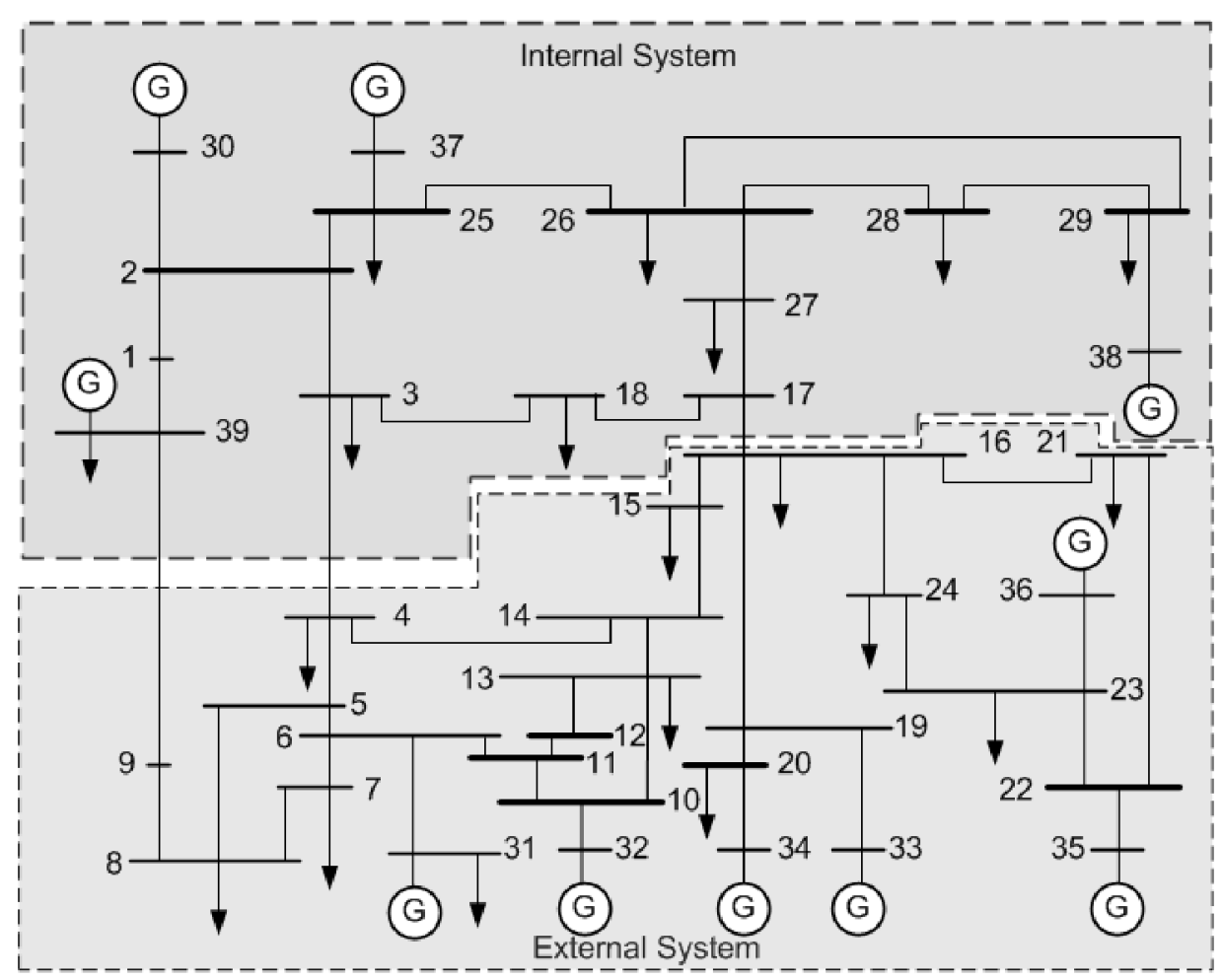

The six generators in the external system are to be aggregated into four equivalent generators after the dynamic reduction. For coherency identification using the proposed method, the Dissimilarity Index (DI) should first be calculated using the rotor angle vectors, which are obtained from the dynamic simulation. The calculated DIs between generators are listed in Table 1. 
Table 1. Dissimilarity Index (DI) table of the test system.

\begin{tabular}{ccccccc}
\hline $\mathbf{D}\left(\boldsymbol{G}_{\boldsymbol{i}}, \boldsymbol{G}_{\boldsymbol{j}}\right)$ & $\mathbf{3 1}$ & $\mathbf{3 2}$ & $\mathbf{3 3}$ & $\mathbf{3 4}$ & $\mathbf{3 5}$ & $\mathbf{3 6}$ \\
\hline 31 & 0 & 0.2622 & 0.3087 & 0.2196 & 0.4577 & 0.5211 \\
32 & 0.2622 & 0 & 0.2637 & 0.2816 & 0.2815 & 0.3807 \\
33 & 0.3087 & 0.2637 & 0 & 0.2002 & 0.3283 & 0.4278 \\
34 & 0.2196 & 0.2816 & 0.2002 & 0 & 0.3868 & 0.4823 \\
35 & 0.4577 & 0.2815 & 0.3283 & 0.3868 & 0 & 0.2118 \\
36 & 0.5211 & 0.3807 & 0.4278 & 0.4823 & 0.2118 & 0 \\
\hline
\end{tabular}

In the table, a smaller value indicates a stronger correlation between two generators, and the index of each generator with itself is equal to zero accordingly. Using these indices, the generators are classified into four coherent groups, based on the PAM algorithm. The generator grouping results are given in Table 2. The representative generators of the groups are determined by the optimal medoids, which are defined during the process of identifying the coherent generator groups using the PAM algorithm. Once the coherent generator groups have been determined, the dynamic reduction is to be completed by aggregating the generators in each group into a representative equivalent generator.

Table 2. Coherency identification result for the proposed algorithm.

\begin{tabular}{cccc}
\hline & Buses & Representative & Sum of DI \\
\hline Group 1 & 31 & 31 & 0 \\
Group 2 & 32 & 32 & 0 \\
Group 3 & 33,34 & 33 & 0.2002 \\
Group 4 & 35,36 & 36 & 0.2118 \\
TC & & 0.4120 & \\
\hline
\end{tabular}

Next, the existing method based on the K-means algorithm is applied to the same IEEE 39-bus test system, and its coherency identification results are listed in Table 3.

Table 3. Coherency identification result of k-mean algorithm.

\begin{tabular}{cccc}
\hline & Buses & Representative & Sum of DI \\
\hline Group 1 & 31 & 31 & 0 \\
Group 2 & 32 & 32 & 0 \\
Group 3 & $33,34,35$ & 33 & 0.5285 \\
Group 4 & 36 & 36 & 0 \\
TC & & 0.5285 & \\
\hline
\end{tabular}

The sums of the dissimilarity indices in each coherent group and the $T C$ are also calculated in this case, and their larger values indicate that coherency identification based on the K-means algorithm does not provide optimized coherent groups for the defined coherency. Comparing these results, one can conclude that the proposed method functions properly, and ensures a more optimized grouping result for the dynamic reduction. 


\subsection{Dynamic Response of the Equivalent Reduced System}

In this section, transient simulations during a system fault are performed for the original system and each of the equivalent reduced systems, and their dynamic responses (the changes in the rotor angles of the generators) are compared. For this purpose, a three-phase line fault is applied to the lines between buses 28 and 29, buses 2 and 3, and buses 17 and 18 at $1 \mathrm{sec}$ respectively, and cleared after six cycles by fault clearing in each case. The rotor angles of the selected generators are then investigated in the original system and both equivalent reduced systems. Figure 5 shows the changes in the rotor angle of a 30-bus generator following each fault.

Figure 5. Rotor angle of a 30-bus generator following each fault.

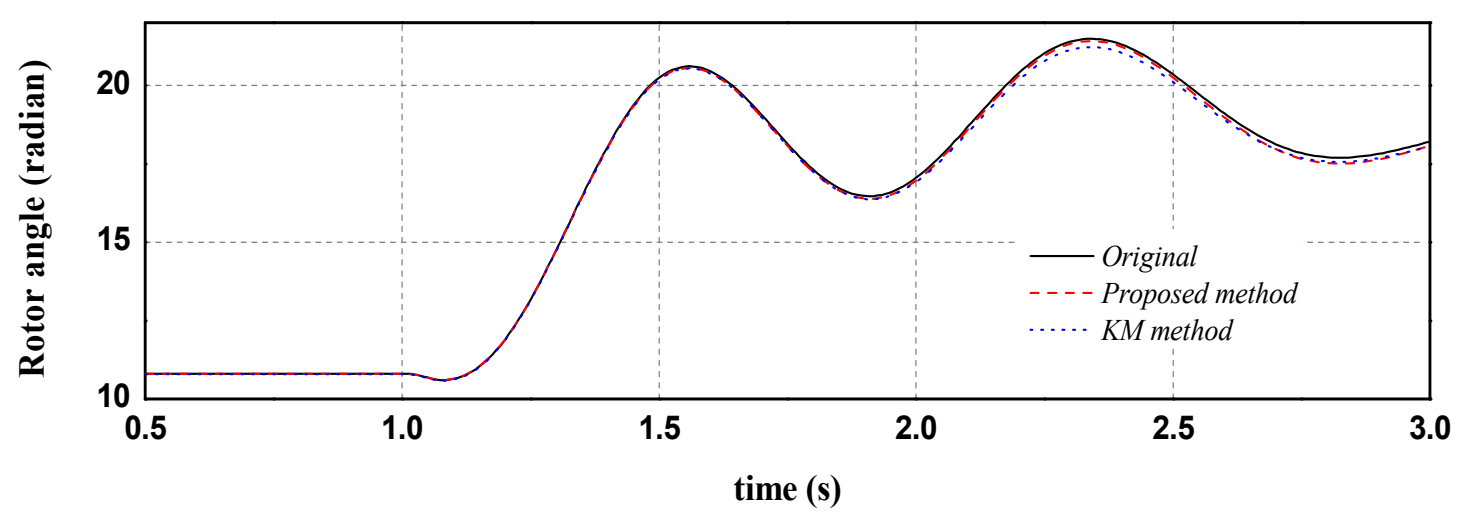

(a) Rotor angle of 30-bus generator following 3-phase line fault at line between 28 and 29 buses.

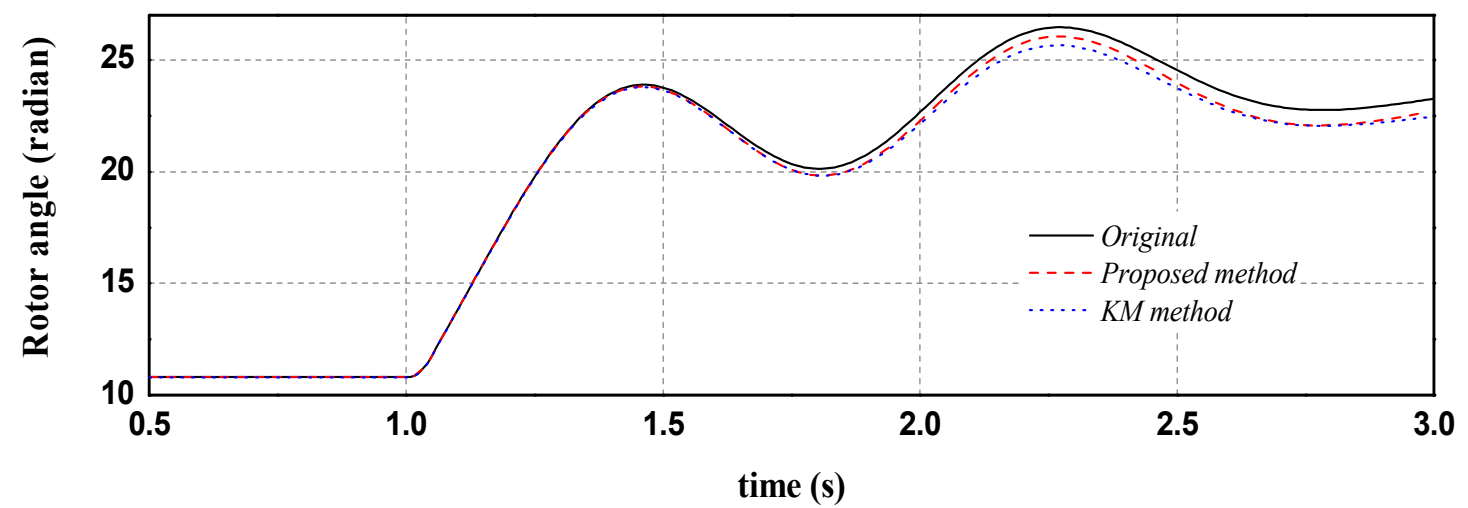

(b) Rotor angle of 30-bus generator following 3-phase line fault at line between 2 and 3 buses.

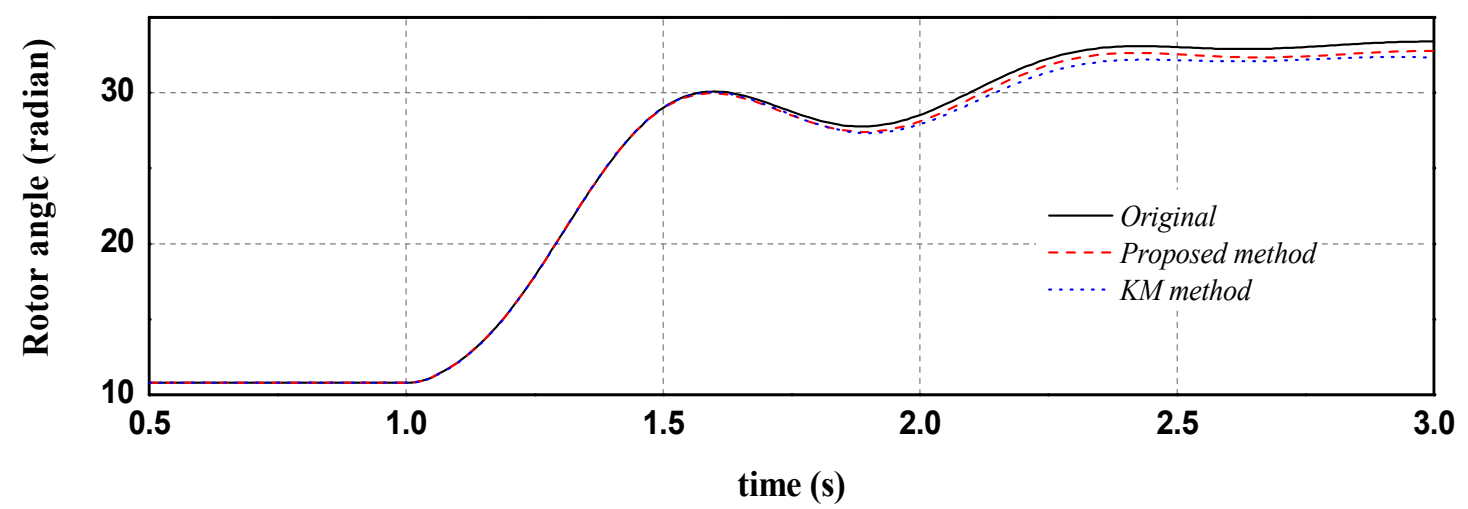

(c) Rotor angle of 30-bus generator following 3-phase line fault at line between 17 and 18 buses. 
As these figures show, the rotor angle patterns of the 30-bus generator in each of the equivalent reduced system (using the proposed technique and the K-means technique) are almost the same as those of the original system. There are some differences between the amplitudes of the swings in the original and reduced systems, but the differences are smaller with the proposed method. This implies that the PAM-based procedure causes less change in the dynamic responses after reduction, and provides a better estimate of the original system. The dissimilarity indices between the same pairs of generators in the original and reduced systems are listed in Table 4, and these reinforce this point more precisely. According to the definition of (4), the DI is defined as the Euclidian distance between the angle vectors of two generators for a given time duration. Therefore, the DI value for the same generators of original and reduced system arithmetically represents the error in the dynamic responses caused by the dynamic reduction. As the table indicates, the DI values for all of the generators are smaller with the proposed algorithm (PAM case), and this means that the proposed coherency identification method ensures a more accurate dynamic reduction compared to the K-means algorithm.

Table 4. Comparison of the Dissimilarity Index (DI) between original and each reduced system.

\begin{tabular}{|c|c|c|c|c|c|c|c|}
\hline \multirow{2}{*}{\multicolumn{2}{|c|}{ Cases }} & \multicolumn{4}{|c|}{ Generators } & \multirow{2}{*}{ Average } & \multirow{2}{*}{ Difference } \\
\hline & & 30-bus & 37-bus & 38-bus & 39-bus & & \\
\hline \multirow{2}{*}{$\begin{array}{l}2-3 \text { line } \\
\text { fault }\end{array}$} & KM case & 0.2806 & 0.2484 & 0.2364 & 0.3148 & 0.2701 & \multirow{2}{*}{0.0782} \\
\hline & PAM case & 0.2081 & 0.1908 & 0.1688 & 0.1999 & 0.1919 & \\
\hline \multirow{2}{*}{$\begin{array}{c}17-18 \text { line } \\
\text { fault }\end{array}$} & KM case & 0.2788 & 0.2071 & 0.2134 & 0.2053 & 0.2262 & \multirow{2}{*}{0.0946} \\
\hline & PAM case & 0.1477 & 0.1097 & 0.1291 & 0.1398 & 0.1316 & \\
\hline \multirow{2}{*}{$\begin{array}{c}28-29 \text { line } \\
\text { fault }\end{array}$} & KM case & 0.1108 & 0.1096 & 0.0936 & 0.1398 & 0.1135 & \multirow{2}{*}{0.0222} \\
\hline & PAM case & 0.086 & 0.0892 & 0.0622 & 0.1278 & 0.0913 & \\
\hline
\end{tabular}

\subsection{Discussion}

Existing studies of dynamic reduction have focused primarily on obtaining an accurate equivalent model for a given set of coherent generator groups, and the development of accurate grouping algorithms for determining the coherent groups has received comparatively little attention. However, since power systems are becoming increasingly complex, the demand for more accurate generator grouping algorithms is increasing. Compared with the existing methods of [12] and [13], the advantages and improvements of the proposed technique can be summarized as follows:

First, optimization using the proposed method is robust, rather than simply providing local optimal results. Use of the PAM algorithm ensures that the coherent grouping results are not influenced by the calculation sequence or the initial conditions in the proposed approach. The PAM algorithm classifies generators into optimal coherent groups minimizing the sum of the dissimilarity indices in each group. In this process, almost all available generator sets are tested as possible medoid sets, and the generator set that minimizes the cost function (TC) is selected. This ensures that an optimized coherent generator set is determined, regardless of the calculation sequence or initial conditions.

Second, additional sequences to determine the representative generators of each group are not necessary in the proposed approach. Once the coherent groups are determined by the coherency identification, the representative generators for the aggregation of each group should be defined. In the 
existing techniques, additional sequences are required to define the representative generator from among the generators in a given group. However, since the proposed method utilizes medoids (the representative object of a group in the PAM algorithm), the representative generators and buses in each group are naturally defined with the medoids.

Finally, since the PAM algorithm examines almost all available cases to determine the optimal medoid set, the execution speed of the proposed method may be slower than that of existing techniques based on sequential processing, especially when the power system is very large and has lots of generator accordingly. To compensate for this weakness, some adjustments are adopted for determining the initial medoid set in the PAM-based approach. Specifically, some parts of the initial medoid set are defined using existing system information, such as the generator configurations of the system. Then, the remainder of the initial medoid set is determined in terms of contributions to objective function, by minimizing the TC. This makes it possible to define the initial medoid set close to the optimal medoid set, and hence the execution speed is improved by reducing the overall number of swapping processes.

\section{Conclusions}

This paper presents a new coherency identification method based on the PAM algorithm, a typical clustering algorithm, which has been used in data mining area. The objective of the proposed coherency identification technique is to determine the optimized coherent groups of generators with respect to the dynamic response. For this purpose, the coherency between generators is first evaluated using the dynamic simulation time response, and is used to define a dissimilarity index. This index provides the criterion for determining the coherent generator groups in the new procedure. Using the PAM algorithm, the coherent generator groups are then determined so that the sum of the dissimilarity indices in each group is minimized. Finally, once the optimal coherent groups have been determined, the generators and buses in the same group are aggregated and replaced by equivalent generators and buses, while retaining the steady-state power flow of the original system in the dynamic reduction. In order to validate the effectiveness of the proposed coherency identification method, simulated cases with an IEEE 39-bus test system were evaluated using PSS/E. The proposed technique was compared with an existing coherency identification method based on the K-means algorithm, and the results show that new procedure provides a better estimate of the original system

\section{Acknowledgments}

This work was supported partially by the New and Renewable Energy Program of the Korea Institute of Energy Technology Evaluation and Planning (KETEP) grant funded by the Korea government Ministry of Knowledge Economy (No.20104010100490) and partially by the MKE (The Ministry of Knowledge Economy), Korea, under the "ITRC" support program supervised by the NIPA (National IT Industry Promotion Agency) (NIPA-2012-C1090-1001-0004). 


\section{References}

1. Newell, R.J.; Risan, M.D.; Allen, L.; Rao, K.S.; Stuehm, D.L. Utility experience with coherency-based dynamic equivalents of very large systems. IEEE Trans. Power Appar. Syst. 1985, 104, 3056-3063.

2. Troullinos, G.; Dorsey, J.; Wong, H.; Myers, J. Reducing the Order of Very Large Power System Models. IEEE Trans. Power Syst. 1988, 3, 127-133.

3. Wang, L.; Klein, M.; Yirga, S.; Kundur, P. Dynamic reduction of large power systems for stability studies. IEEE Trans. Power Syst. 1997, 12, 889-895.

4. William, W. Large-Scale System Testing of a Power System Dynamic Equivalencing Program. IEEE Trans. Power Syst. 1998, 13, 768-774.

5. Lei, X.; Povh, D.; Ruhle, O. Industrial approaches for dynamic equivalents of large power systems. Proc. Power Eng. Soc. Winter Meet. 2002, 2, 1036-1042.

6. Kim, H.; Jang, G.; Song, K. Dynamic reduction of the large-scale power systems using relation factor. IEEE Trans. Power Syst. 2004, 19, 1696-1699.

7. Yang, J.P.; Cheng, G.H.; Xu, Z. Dynamic Reduction of Large Power System in PSS/E". In Proceedings of the 2005 IEEE/PES Transmission and Distribution Conference and Exhibition: Asia and Pacific, Dalian, China, August 2005; pp. 1-4.

8. Robin, P. Identification of Coherent Generators for Dynamic Equivalents. IEEE Trans. Power Appar. Syst. 1978, 1, 1344-1354.

9. Giri, J.C. Coherency reduction in the EPRI stability program. IEEE Trans. Power Appar. Syst. 1983, 102, 1285-1293.

10. Pires de Souza, E.; Leiti da Silva, A. An efficient methodology for coherency-based dynamic equivalents. IEE Proc. 1992, 139, 371-382.

11. Ourari, M.L.; Dessaint, L.A.; Van-Que, D. Dynamic equivalent modeling of large power systems using structure preservation technique. IEEE Trans. Power Syst. 2006, 21, 1284-1295.

12. Van Oirsouw, P.M. A Dynamic Equivalent Using Modal Coherency and Frequency Response. IEEE Trans. Power Syst. 1990, 5, 289-295.

13. Kim, J.Y.; Won, D.J.; Moon, S.I. A Study on the Dynamic Reduction for Large Power System. KIEE Int. Trans. 2002, 12, 1-5.

14. Joo, S.K.; Liu, C.C.; Jones, L.E.; Choe, J.W. A Coherency and aggregation techniques incorporating rotor and voltage dynamics. IEEE Trans. Power Syst. 2004, 19, 1068-1075.

15. Spath, H. Cluster Analysis Algorithms; Halsted Presses: New York, NY, USA, 1980.

16. Machowski, J.; Cichy, A.; Gubina, F.; Omahen, P. External Subsystem Equivalent Model for Steady-state and Dynamic Security Assessment. IEEE Trans. Power Syst. 1988, 3, 1456-1463.

(C) 2012 by the authors; licensee MDPI, Basel, Switzerland. This article is an open access article distributed under the terms and conditions of the Creative Commons Attribution license (http://creativecommons.org/licenses/by/3.0/). 